\title{
Scale-free topologies and activatory-inhibitory interactions
}

\author{
J. Gómez-Gardeñes \\ Departamento de Física de la Materia Condensada, Instituto de Biocomputación y Física de Sistemas \\ Complejos BIFI, and Departamento de Teoría y Simulación de Sistemas Complejos, Instituto de Ciencia \\ de Materiales de Aragón ICMA, C.S.I.C.-Universidad de Zaragoza, 50009 Zaragoza, Spain \\ Y. Moreno ${ }^{\text {a) }}$ \\ Departamento Física Teórica and Instituto de Biocomputación y Física de Sistemas Complejos BIFI, \\ University of Zaragoza, 50009 Zaragoza, Spain \\ L. M. Floría \\ Departamento de Física de la Materia Condensada, Instituto de Biocomputación y Física de Sistemas \\ Complejos BIFI, and Departamento de Teoría y Simulación de Sistemas Complejos, Instituto de Ciencia \\ de Materiales de Aragón ICMA, C.S.I.C.-Universidad de Zaragoza, 50009 Zaragoza, Spain
}

(Received 30 August 2005; accepted 27 October 2005; published online 31 March 2006)

\begin{abstract}
A simple model of activatory-inhibitory interactions controlling the activity of agents (substrates) through a "saturated response" dynamical rule in a scale-free network is thoroughly studied. After discussing the most remarkable dynamical features of the model, namely fragmentation and multistability, we present a characterization of the temporal (periodic and chaotic) fluctuations of the quasi-stasis asymptotic states of network activity. The double (both structural and dynamical) source of entangled complexity of the system temporal fluctuations, as an important partial aspect of the correlation structure-function problem, is further discussed in light of the numerical results, with a view on potential applications of these general results. (c) 2006 American Institute of Physics.
\end{abstract}

[DOI: $10.1063 / 1.2146115$ ]

Many real networks are complex and heterogeneous. In this paper, we study a dynamics that generically describes biological processes that take place on complex architectures as metabolic reactions and gene expression. We capitalize on the theory of nonlinear dynamical systems to uncover the topological and dynamical patterns of a Michaelis-Menten-type dynamics coupled to a network that is complex, directed, and highly skewed. The results indicate that such patterns can exist in the form of periodic and chaotic orbits revealing interesting properties at both local and global levels. Moreover, the dynamics on top of the substrate networks yields topologically complex substructures (islands or clusters) whose structural characteristics are analyzed. This analysis offers interesting results on the interplay structure-function. We round off our study by discussing possible implications of heterogeneous topologies on biological processes at the cellular level.

\section{INTRODUCTION}

Nonlinear lattices, i.e., spatially discrete many-body systems with nonlinear interactions, are currently the subject of considerable multidisciplinary interest, not only among a wide variety of Physics subdisciplines and technologies, ${ }^{1}$ but also in Biomolecular Chemistry, Cell Physiology, Theoretical Biology, Social Sciences, and other fields. ${ }^{2}$ There is a common basic interest in the understanding of the many aspects of the correlation between structure and function in systems

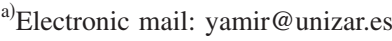

made up of discretely many nonlinearly interacting components.

While in Physics applications the interactions among constituents (atoms, magnetic moments, etc.) usually depend on the geometrical distance between their positions in real space, in applications outside "fundamental physics" the space of interactions is abstracted, so that proximity between components (say $i$ and $j$ ) is measured as the length of the path of interactions given by a connectivity matrix $C_{i j}$. In other words, the graph (or network) of interactions, and not anymore the real space, fixes the relevant geometry related to the function (dynamics) of the system. This does not preclude the applicability of statistical and field theory methods to the study of nonlinear lattices outside the traditional physics subdisciplines; on the contrary, a proper use of these approaches often throws considerable light on some important issues currently addressed. ${ }^{3}$

Though lattice disorder effects on nonlinear dynamics of macroscopic systems have their own tradition, the most usual case in physics is that of homogeneous (either pure random or regular) networks. However, recent confluent studies on the structure of interactions in a large variety of technological (communication, power grid) as well as biomolecular (protein-protein interaction, gene regulation, cell metabolism), ecological (trophic networks, mutualism) and socioeconomic systems have shown the overabundance of highly inhomogeneous structures ${ }^{4,5}$ among "real world" interacting systems.

Homogeneity of the interactions structure means that almost all nodes are topologically equivalent, like in a regular lattice or in a random Erdös-Renyi graph, thus showing a 


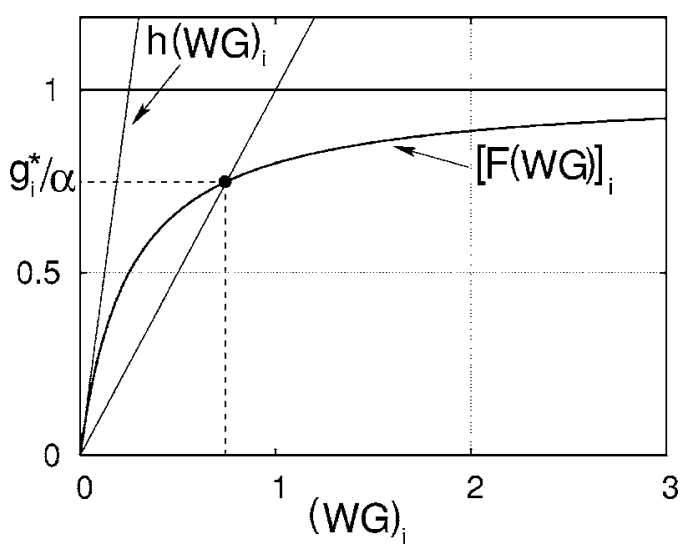

FIG. 1. Saturated functional response $(h=4)$.

density distribution function of the degree of connectivity localized around a mean value with a well-defined average of quadratic fluctuations. If $k$ denotes the degree (number of interactions of a given node) and $P(k)$ denotes its density distribution function, an inhomogeneous network shows for $P(k)$ a power law (often truncated). The absence of a characteristic scale in the connectivity patterns (scale-free networks) manifests itself in the presence of a small number of nodes (named hubs) connected to very many nodes, and a larger number of poorly connected nodes. The complex character of the structure of the interactions couples to the dynamical complexity which emerges from the nonlinear character of the interactions, so that generally one may say that the structure-function correlation problem in real networks has at least two sources of entangled complexity.

The model that we analyze is introduced in Sec. II. It tries to capture general ingredients of this entangled complexity in a relevant kind of nonlinear dynamics: Activation/ inhibition (AI) competing interactions with a "saturated response" rule for the rate of activation (see Fig. 1). This kind of dynamics is often called Michaelis-Menten, ${ }^{6}$ Holling, ${ }^{7}$ or Langmuir ${ }^{8}$ rule. The interacting units sit on a lattice which is both small-world (i.e., short mean path length) and scalefree. For this we use the Barabási-Albert ${ }^{9}$ network. Afterwords, some basic general features of the model are discussed, namely the network fragmentation in subclusters (or islands) of collective dynamics (Sec. II A), and the generic types of asymptotic behaviors coexisting in the phase space of collective dynamics as well as the observed bifurcations in phase portrait upon parameter variations (Sec. II B). These basic consequences of the AI competition on the complex network are prevalent for values of the ratio AI ranging from 1 to 6. Finally, in Sec. II C the bifurcations found are explained in terms of the Floquet analysis of the solutions.

Section III mainly reports on the statistical characterization of both the dynamical behaviors observed (Sec. III A) and the structural characterization of the dynamical islands (Sec. III B). We perform an extensive exploration of the parameter space, employing different initial conditions and substrate network realizations, in order to find the conditions for the existence of chaotic and periodic behavior as well as to fully characterize the main topological characteristics of the dynamical islands. Finally, in Sec. III C we identify those substructures of the dynamical islands that are relevant for the dynamical evolution of the system.

The concluding Sec. IV summarizes the main conclusions of our work, along with some prospective remarks on likely applications of the model, and the potential use of these techniques in the study of particularly interesting realworld biological networks.

\section{THE MODEL. BASIC DYNAMICAL FEATURES}

As stated in Sec. I, here we introduce a model of activatory/inhibitory interactions regulating the activity $g_{i}(t)$ $(i=1, \ldots, N)$, of $N$ constituents (e.g., agents, substrates), with $N$ generally being a large number. The real functions of time $g_{i}(t)$ are each attached to a node of a graph with adjacency matrix $C_{i j}(N \times N)$. The matrix element is nonzero, $C_{i j} \neq 0$, only if the rate of variation of the $i$ th node activity, $g_{i}(t)$, depends on the activity $g_{j}$ of the $j$ th node (interaction $i \leftarrow j$ ). Different realizations of the $C_{i j}$ matrix are constructed using the method of Barabási and Albert, ${ }^{9}$ in order to ensure two seemingly universal characteristics of many recently studied networks in biological and social sciences and other fields: ${ }^{4,5}$

(a) Small-world, meaning that the mean distance (minimal length of the interaction path), $\left\langle l_{i j}\right\rangle$, between pairs of nodes goes at most as $\log N$, for large values of $N$.

(b) Scale-free, meaning that the density distribution function $P(k)$ of the degree (connectivity) of nodes scales as $P(k) \sim k^{-\gamma}$, with $\gamma=3$. Other values of $\gamma(2 \leqslant \gamma$ $\leqslant 3$ ) were also analyzed by using suitably tested modifications of the Barabási-Albert preferential attachment rule. $^{10}$

The interaction $(i \leftarrow j)$ can be either activatory (excitatory) or inhibitory; correspondingly we define the interaction matrix element $W_{i j}$ to be +1 or -1 , respectively (and $W_{i j}$ $=0$ whenever $C_{i j}=0$ ), and call $p$ the fraction, among nonzero elements, of negative signs (note that while $C_{i j}$ is a symmetric matrix, $W_{i j}$ is not in general). Moreover, the sign distribution of elements is taken uniform in the set of (approximately $\langle k\rangle N / 2$ ) links of the network realization.

The dynamics of the nodes activity vector $\mathbf{G}(t)=\left\{g_{i}(t)\right\}$ (with $i=1, \ldots, N$ ) that we consider is such that only in the presence of excitatory neighbors activity could $g_{i}$ possibly be non-null, otherwise $g_{i}$ decays to zero with an exponential rate:

$$
\frac{d \mathbf{G}(t)}{d t}=-\mathbf{G}(t)+\alpha \mathbf{F}(\mathbf{W G}(t)),
$$

where $\mathbf{F}$ is a nonlinear vector function whose argument is the product of the interaction matrix $\mathbf{W}$ and the activity vector $\mathbf{G}$, and $\alpha(>0)$ accounts for the strength of the interaction. The function $\mathbf{F}$ is a saturated response function (see Fig. 1), defined as

$$
\mathbf{F}(\mathbf{z})=\left\{\frac{\Phi\left(z_{i}\right)}{h^{-1}+\Phi\left(z_{i}\right)}\right\},
$$

where $\Phi(x)$ is a function defined as $\Phi(x)=x$ if $x \geqslant 0$ and zero otherwise. In our numerical studies of the model we have fixed the value of the parameter $\alpha=3$, and varied the param- 

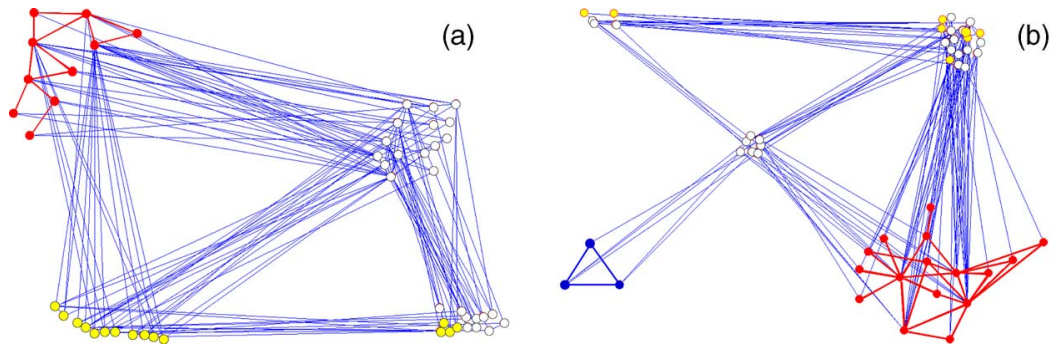

(b)

FIG. 2. (Color online) Two examples of network fragmentation. The nodes of the networks are classified in: (i) dynamical nodes (red), (ii) stationary nodes with nonzero activity (blue), (iii) stationary nodes with zero activity belonging to $\mathcal{D}_{0} \oplus \mathcal{D}_{1}$ (yellow), and (iv) remaining nodes with zero activity (white). Note that the white central nodes in (b) act as the frontier between the dynamical island and the steady nonzero activity one.

eters $0 \leqslant p \leqslant 1$ and $0 \leqslant h \leqslant 10$. One can easily realize that the solutions for non-negative initial conditions remain bounded for all $t \geqslant 0$. Indeed, as the nonlinear term in Eq. (1) is bounded above by $\alpha$, one obtains that $\dot{g}_{i}<0$ whenever $g_{i}$ $>\alpha$. Also, if $g_{i}=0$ then $F_{i}(\mathbf{W G}) \geqslant 0$, so that the activities cannot become negative.

The above-noted dynamics can be regarded, e.g., as a generalization of some simplified and coarse-grained genetic models, referred to as random Boolean networks, ${ }^{11}$ where Boolean rules are implemented. These have been extensively used to study networks at various levels of biological organization, and have provided useful insights later supported by experiments. ${ }^{11,12}$ Equation (1) incorporates the experimental observation of a continuous range of activity levels. ${ }^{13}$ While linear models have been successful for the reconstruction of the interaction networks from experimental data, ${ }^{14}$ nonlinear models like Eq. (1) are expected to be more appropriate for a quantitative description of the dynamics.

The dynamics (1) of a two-agent (dimer) model has been considered in Ref. 13, in the context of virus-cell interactions in bacteria and general gene regulatory activity models, where a rich repertoire of behaviors, like multistability (multiple attractors in phase space) was reported. A preliminary study of the behavior of Eq. (1) on small-world scale-free networks can be found in Ref. 15, where the interested reader can find a more detailed account of the numerical techniques used in the characterization of the different dynamical regimes. In the following, we review some remarkable general features of the network dynamics.

\section{A. Activation and inhibition interplay: Fragmentation}

A brief look at Eq. (1) easily reveals that for any value of the parameters $p$ and $h$ the state of inactivity, $\mathbf{G}=0$, is always a solution. As a matter of fact, for $h=0$, or $h \neq 0$ but $p=1$, this is the unique asymptotic solution (global attractor in the phase space) for all possible non-negative initial conditions. However, for $h \neq 0$ and $p \neq 1$ other asymptotic solutions, with islands of positive activity, generically coexist with the rest state. The term islands denotes here subnetworks that are interconnected through nodes which have evolved to null activity, so that their dynamics are effectively disconnected.

The fragmentation of the network dynamics into disconnected islands is a generic feature of AI interactions, as the following considerations suggest. Let us call $\mathcal{D}$ the set of nodes whose activities, for a given initial condition $\mathbf{G}(t=0)$, asymptotically vanish. It is easy to see that, irrespective of the initial condition, this set is generically nonempty.
Indeed, if a node $i$ is such that $W_{i j}=-1$ or 0 for all $j$, then its activity $g_{i}(t)$ will tend to zero. Let us call $\mathcal{D}_{0}$ the set of these nodes, and note that its measure $\left(\Sigma_{k} P(k) p^{k}\right)$ is a nonzero increasing function of $p$. Now, call $\mathcal{D}_{1}$ the set of nodes $l$ such that their positive $W_{l j}$ occur for $j$ 's in $\mathcal{D}_{0}$, and so on. Due to the small-world property, there are in fact very few relevant $\mathcal{D}_{n}(n=0,1, \ldots)$ sets. Its union $\mathcal{D}^{*}=\cup \mathcal{D}_{n}$ is easily seen to have a nonzero measure which increases with $p$.

The nodes of $\mathcal{D}^{*}$ are structurally (i.e., irrespective of initial conditions) inactive. Depending on the initial condition, the set $\mathcal{D}$ may include other nodes not contained in $\mathcal{D}^{*}$, namely those nodes that evolve to inactivity due to the initial condition (dynamically, instead of structurally, inactive): See, e.g., the white nodes in Fig. 2, where we show two small networks of $N=50$ nodes to allow a simple visualization of the sets $\mathcal{D}^{*}$ and $\mathcal{D}$. In other words, the measure of $\mathcal{D}$ may in general be (much) larger than the measure of the "structurally dead" nodes $\mathcal{D}^{*}$.

From the previous considerations, whether or not the set $\mathcal{D}$ percolates the network realization, leaving out islands of disconnected activity, is an event that clearly depends on both the parameter $p$ and the initial conditions. But also the discussion correctly suggests that fragmentation of the network into subclusters with independent temporal evolution is a generic (nonzero measure) feature. Our numerics, which are extensive in the sense of (both network realizations and initial conditions) large sampling, convincingly corroborate this assertion. Figures 3 and 4 show two islands of periodic and chaotic activity, respectively, as well as the temporal evolution of $g_{i}(t)$ for some of their constituent's nodes (see the next section for a more detailed discussion of the figures).

\section{B. Temporal fluctuations of asymptotic solutions}

The asymptotic dynamics of Eq. (1) was studied in Ref. 15. We summarize here the most salient features of the phase portrait of the collective dynamics.

The presence of inhibitory interactions makes possible the existence of instabilities in the fixed point solutions [i.e., states of constant activities, $g_{i}(t)=g_{i}^{*}$, let us say chemostasis regime] of evolution equations (1). Using linear stability analysis techniques, these "typical" instabilities are characterized as Hopf bifurcations (either direct or often inverse), where attractors of exactly periodic collective activities, $g_{i}(t)=g_{i}(t+T)$, are born out from the unstable fixed points. The inverse period (frequency) $\omega=1 / T$ of a periodic attractor changes with parameter and is naturally dependent on each specific island realization. A sampling over different initial 

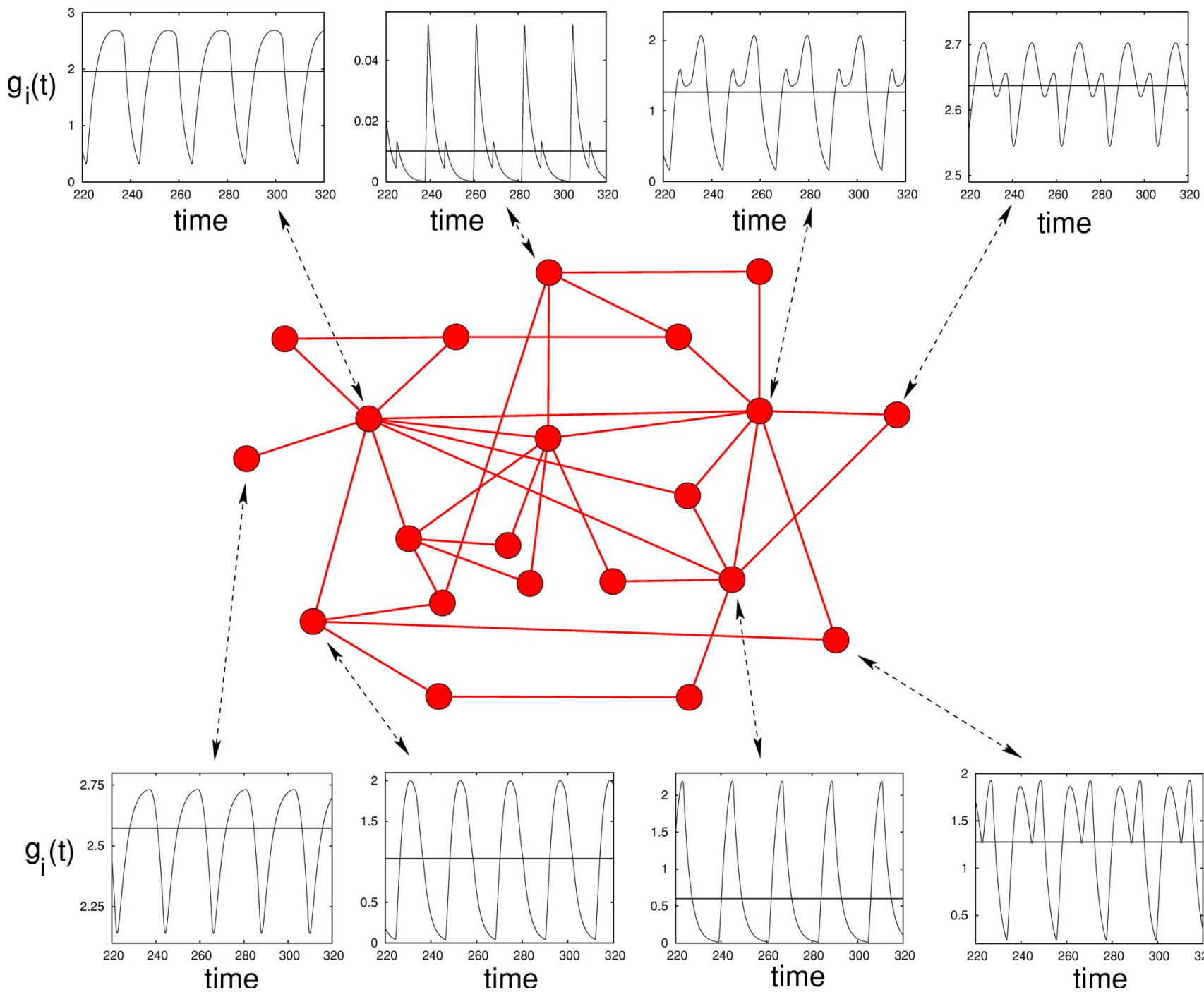

FIG. 3. (Color online) Example of a cluster of 21 nodes displaying periodic dynamics. The insets show the dynamical patterns of each node (see the text for the interpretation). The maximum Liapunov exponent is $\lambda=-0.00034$ and the dynamical parameters are $h=4$ and $p=0.7$. The cluster is embedded in a substrate network of $N=50$.

conditions and network realizations shows that $\omega$ is smoothly peak-distributed around a value of order unity (the characteristic time scale of activity decay in the absence of interactions) with a decaying queue slightly biased to higher frequency values. ${ }^{15}$

One easily observes that these periodic attractors, in turn, also typically experience period doubling instabilities, and through the well-known universal scenario of (successive) period doubling bifurcation cascade, the onset of chaotic attractors takes place in the phase portrait of the network dynamics. To help visualization of the generic types of asymptotic network dynamics, we represent in Fig. 5 the bifurcation diagram for a typical attractor. At different values of the (Michaelis-Menten) parameter $h$, and constant values of $\alpha=3, p=0.7$, we plot the activity of an individual node at the instant when its time derivative vanishes. Thus, a single branch in the figure indicates stationary activity, two branches indicate a periodic attractor, etc. We also plot in Fig. 5 the largest Lyapunov exponent $\lambda$ on the attractor, so to allow discerning between chaotic (positive $\lambda$ ) and eventual regular quasiperiodic evolutions $(\lambda=0)$.
A similar bifurcation diagram for a different network realization is shown in Fig. 6, where one can appreciate (see the inset) a commonly found bifurcation (though it appears much less often than period doubling), namely period tripling bifurcation. Its characterization will be made in Sec. II $\mathrm{C}$ where the Floquet analysis of periodic attractors is presented.

To illustrate the aspect of typical periodic fluctuations we show in Fig. 3 some examples of the temporal activity $g_{i}(t)$ of different nodes inside an island of synchronized activity from a representative system. Note that the abundance of out of phase oscillations of neighbors activity is a natural consequence of inhibitory interactions. Horizontal lines in insets indicate the average level $\bar{g}_{i}$ of node activity. We see that in some of the island nodes the amplitude of the oscillation is small compared to $\bar{g}_{i}$ (see, e.g., top rightmost and bottom leftmost insets); while in others they are of comparable size, even to the point that lowest levels of activity can reach a null value, before activity is triggered again after inhibiting neighbors activity decreases enough. 


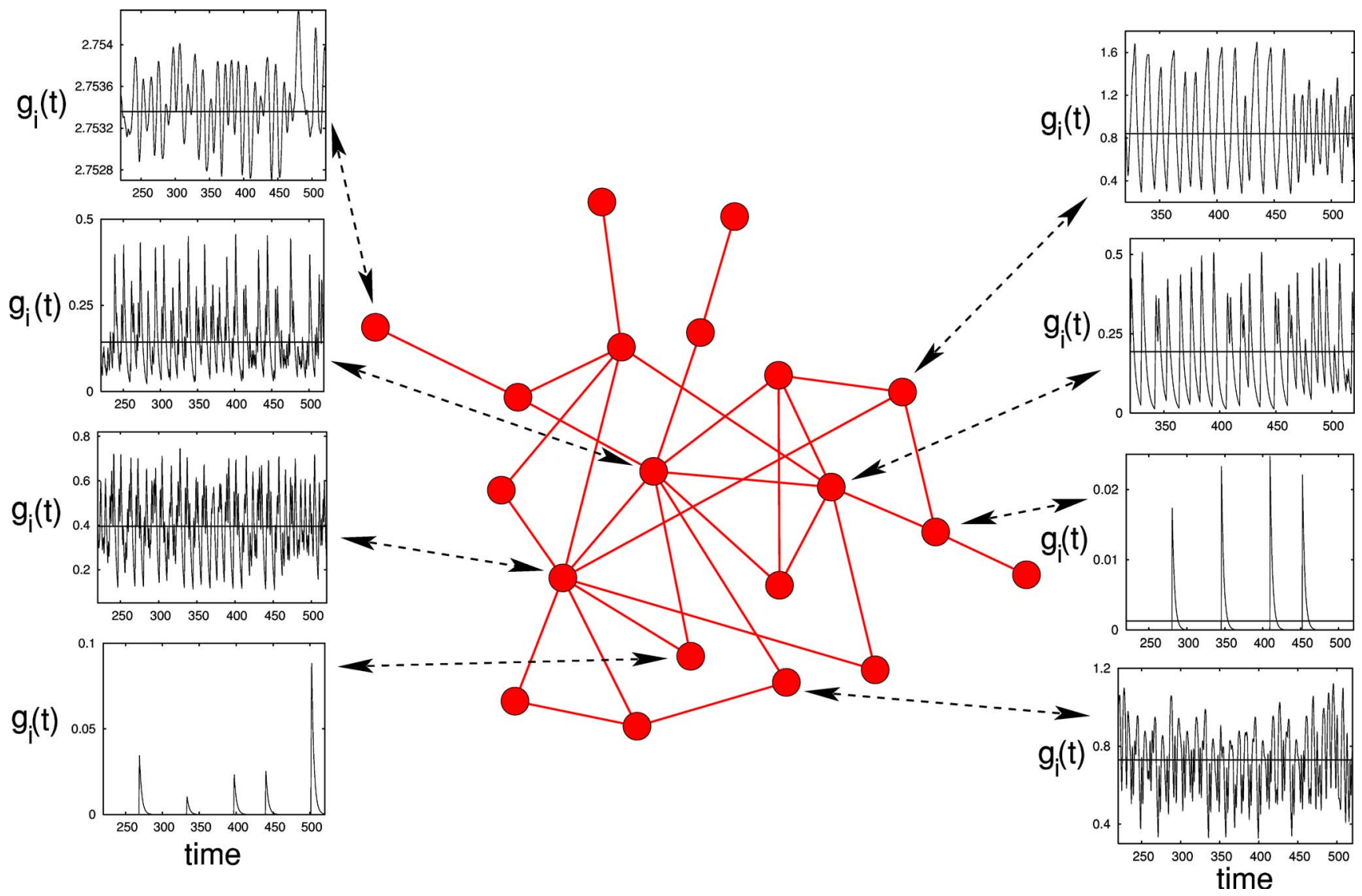

FIG. 4. (Color online) Example of a cluster of 19 nodes displaying chaotic dynamics. The insets show the dynamical patterns of each node (see the text for the interpretation). The maximum Liapunov exponent is $\lambda=0.4716$ and the dynamical parameters are $h=4$ and $p=0.7$. The cluster is embedded in a substrate network of $N=50$.

An analogous visualization of chaotic temporal fluctuations of the activities in a cluster is shown in Fig. 4. Here again we see nodes (e.g., top left inset) where the size of activity fluctuations is less than $1 \%$ of the average level $\bar{g}_{i}$. Most remarkable, there are nodes (like the one in bottom left inset) which remain inactive most of the time intermittently experiencing spikes of short duration activity. This amazing variability of individual node temporal activity on the chaotic attractors is a generic feature of the network dynamics. The existence of spike behavior of individual nodes' activity suggests correctly that eventual variations of parameters like $h$ may lead to permanent inactivity of some particular nodes, so providing a straightaway decreasing of the dynamical cluster size or, the other way around, the activation of inactive nodes in the frontier.

It is important to note that, for a fixed set of parameter values and a given network realization, there are generally several different attractors coexisting in the phase space portrait of the network dynamics, each one having its own basin (of attraction) of initial conditions. Multistability appears as a generic consequence of the excitatory/inhibitory interplay. Importantly also, there can be very many unstable periodic trajectories (often entangled) flowing in between basins of attractions. The excitatory/inhibitory competition is also responsible for the appearance of temporally complex (positive Lyapunov exponent) aperiodic evolutions, associated to the bifurcation cascade scenario. As we will show in Sec. III A the manifestation of fluctuating (either periodic or chaotic) temporal behaviors takes importance when inhibitory links predominate, though not too much, over excitatory ones.

\section{Floquet analysis of the periodic attractors}

As shown in the bifurcation diagrams of Figs. 5 and 6, periodic solutions of the network dynamics often become unstable under variations of the model parameters. In order to characterize these instabilities in a precise manner, one may perform the linear stability analysis of the periodic orbits (see, e.g., Ref. 16) near the bifurcation points.

For this we consider small perturbations of the dynamical variables, $\delta \vec{g}\left(t_{0}\right)=\left\{\delta g_{i}\left(t_{0}\right)\right\}$, and compute their evolution over the period $T$ of the periodic orbit. The evolution of these small perturbations (vectors in tangent space) follows the (linear) dynamics obtained by linearizing Eq. (1) around the periodic orbit $\left\{\hat{g}_{i}(t)\right\}=\left\{\hat{g}_{i}(t+T)\right\}$, i.e.,

$$
\frac{d \delta \vec{g}(t)}{d t}=-\delta \vec{g}+\alpha \cdot \mathcal{A} \delta \vec{g},
$$

where the matrix $\mathcal{A}$ is obtained as

$$
\mathcal{A}_{i, j}=\frac{\Theta\left[\Sigma_{k} W_{i, k} g_{k}\right]}{\left(1+h^{-1} \Phi\left[\Sigma_{k} W_{i, k} g_{k}\right]\right)^{2}} \cdot W_{i, j}
$$

and $\Theta[x]$ denotes the (Heaviside) step function. Note that Eq. (4) is only valid when the sum of the inputs (activatories and 


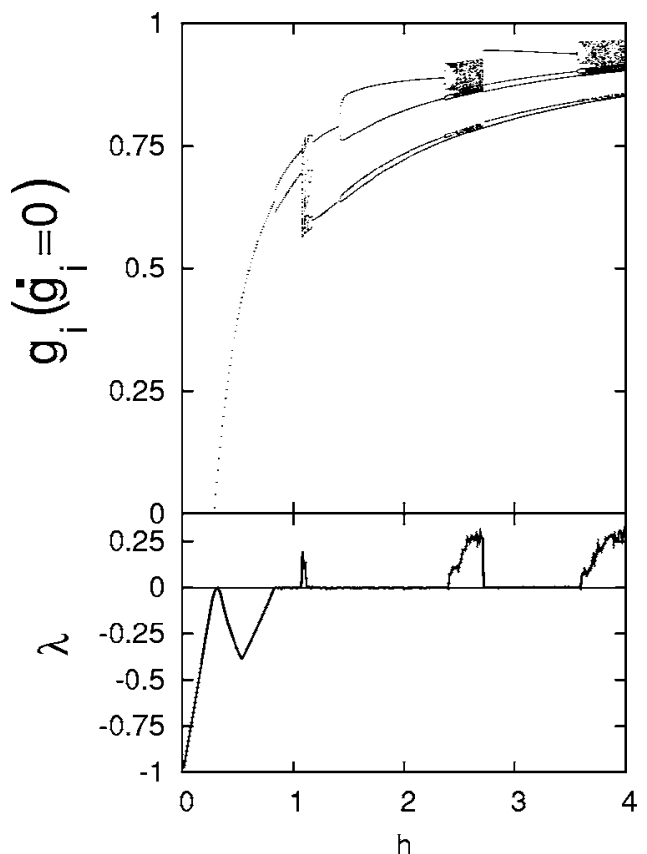

FIG. 5. Example of bifurcation diagram (island size: $14 ; N=60 ; p=0.8$ ). One can appreciate an inverse Hopf bifurcation and several (direct and inverse) period doubling bifurcation cascades. The maximum Lyapunov exponent $\lambda$ is plotted in the lower part.

inhibitories) which receives a node from its neighbors is nonzero. Hence, the Floquet analysis is performed for each dynamical cluster found and not for the whole network.

The so-called Floquet (or monodromy) matrix $\mathcal{F}$ of the periodic solution $\left\{\hat{g}_{i}(t)\right\}$ is defined as the linear operator in tangent space that maps the initial perturbation at $t_{0}, \delta \vec{g}\left(t_{0}\right)$, onto the perturbation at $t_{0}+T$,

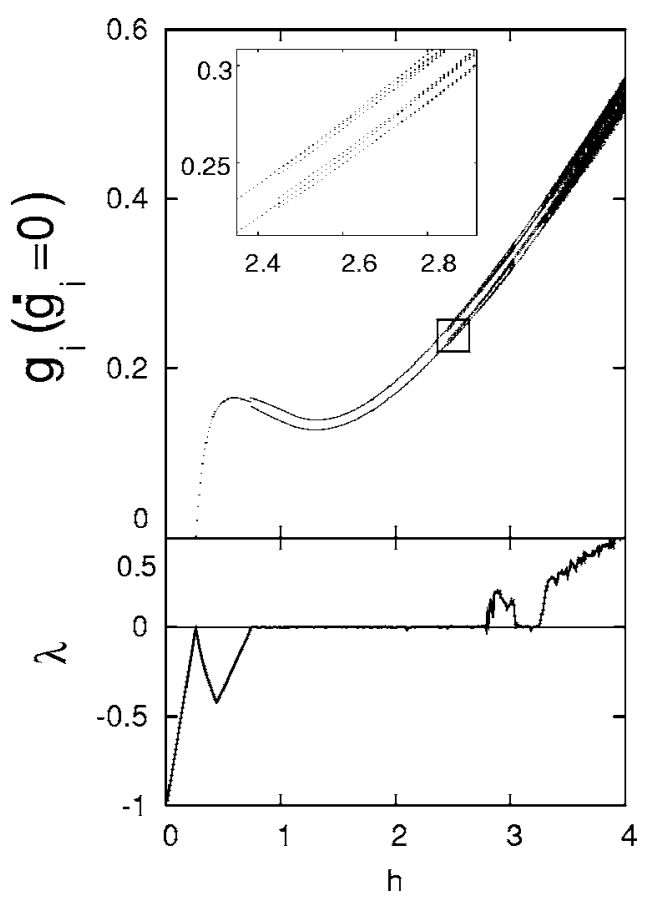

FIG. 6. Example of bifurcation diagram (island size: $12 ; N=60 ; p=0.8$ ) showing (see the inset) a period tripling bifurcation. The maximum Lyapunov exponent $\lambda$ is plotted in the lower part.

$$
\delta \vec{g}\left(t_{0}+T\right)=\mathcal{F} \delta \vec{g}\left(t_{0}\right) .
$$

The Floquet matrix $\mathcal{F}$ is obtained by numerical integration of the linearized Eq. (3) over a period $T$ for a basis of initial conditions in the tangent space. The spectrum of eigenvalues of this matrix provides the information on the linear stability of the periodic solution. Note that because $\mathcal{F}$ is a real matrix, if a Floquet eigenvalue $\mu$ is a complex number, then its complex conjugate $\bar{\mu}$ also belongs to the Floquet spectrum. Also, because solutions of autonomous differential equations can be shifted in the time $t$ direction, their Floquet matrix always has unity as an eigenvalue, say $\mu^{1}=1$, with associated eigenvector $\left\{\dot{\hat{g}}_{i}\left(t_{0}\right)\right\}$. The solution is linearly stable if all the other eigenvalues $\mu^{j}=\left|\mu^{j}\right| \exp \left(i \theta^{j}\right)$ are in the interior of the unit circle of the complex plane, i.e., $\left|\mu^{j}\right|<1$ for $j$ $\neq 1$. A periodic solution becomes unstable when a Floquet eigenvalue (or a pair of complex conjugate eigenvalues) crosses the unit circle. The associated Floquet eigenvector indicates the direction in tangent space where perturbations will grow exponentially away from the solution.

In Fig. 7(a) we plot the Floquet spectrum of a periodic attractor at a period doubling bifurcation. As seen in the figure, a Floquet eigenvalue crosses the unit circle at -1 . In Fig. 7(b) we plot the Floquet spectrum of the periodic attractor of Fig. 6 at $h=2.44$, where the inset suggested that a period tripling bifurcation may occur. We see a complex conjugate pair of Floquet eigenvalues exiting the unit circle at angles $\theta= \pm 2 \pi / 3$. In general, for generic irrational values of $\theta / \pi$ this type of bifurcation (called Naimark-Sacker or general-

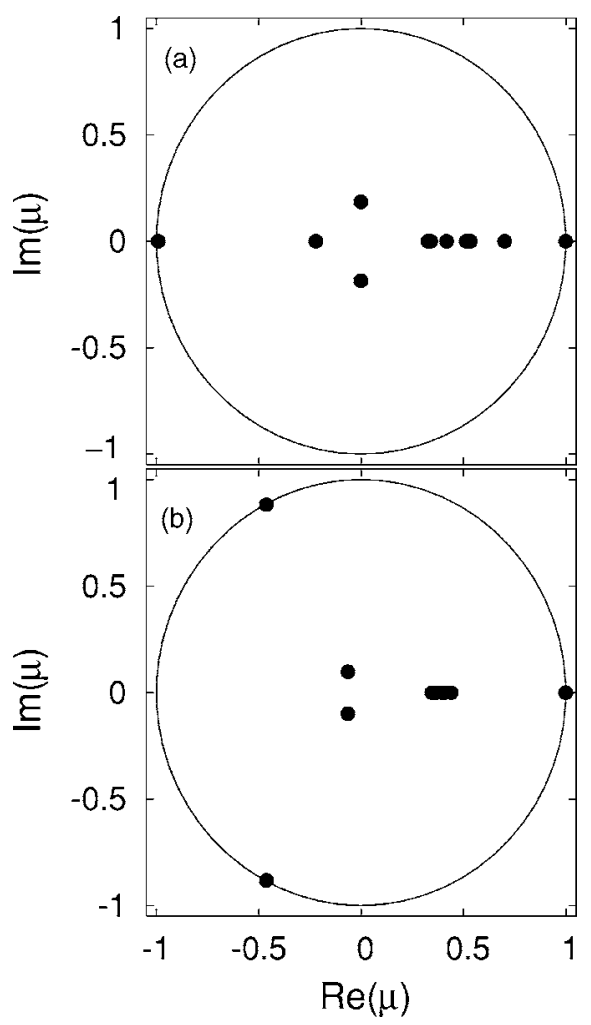

FIG. 7. Floquet spectra. (a) Period doubling bifurcation in an island of size 14 at $h=1.57$. (b) Naimark-Sacker bifurcation (at rational Floquet angle $\theta$ $= \pm 2 \pi / 3$ ) in an island of size 12 at $h=2.44$ (the same as used in the diagram of Fig. 6). For both $N=60$ and $p=0.8$. 


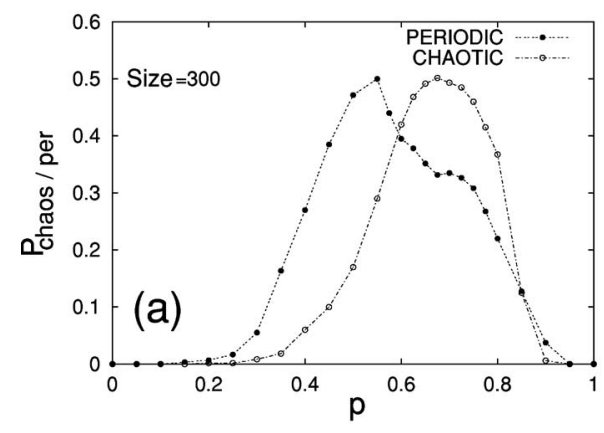

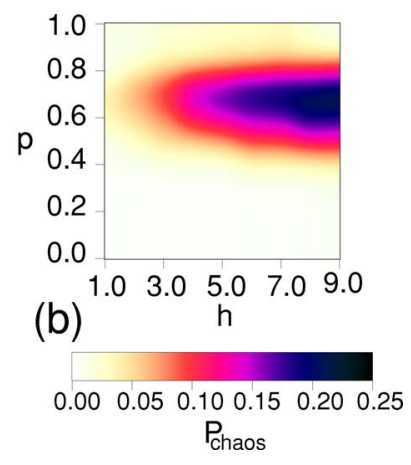

FIG. 8. (Color online) (a) Probability, $P_{\text {chaos }}\left(P_{\text {per }}\right)$, that the system evolves to a chaotic (periodic) regime as a function of the probability of inhibitory interactions, $p$, for $h=4$ and $N=300$. (b) Phase diagram in the $(p, h)$-parameter space of the chaotic dynamics of the system. Color code indicate the values of $P_{\text {chaos }}(N$ $=300$ ). ized Hopf bifurcation) gives rise to a quasiperiodic attractor whose trajectories fill densely a two-frequency torus. However, as a generic feature of our model, the two frequencies of the new attractor are in a commensurate ratio (2:3), so that the new stable trajectory has a period of $3 \mathrm{~T}$.

In terms of how often different types of bifurcation occur in the network dynamics, as inferred from our (nonexhaustive, but significant at the scales considered) sampling of initial conditions and network realizations, one may say that period doubling cascades and, less often, commensurate Naimark-Sacker bifurcations have been generically found by varying the Michaelis-Menten parameter $h$. But, besides the formal characterization of the dynamical instabilities observed, the Floquet analysis allows one also to give an answer on a more general question, namely how temporal instabilities correlate with networking connectivity characteristics. Are there characteristic features discernible in the structure of instabilities? This point will be discussed further.

\section{STATISTICAL CHARACTERIZATION OF DYNAMICAL REGIMES AND ISLANDS STRUCTURE}

As noted before, the dynamics of the system is determined by only two parameters, $h$ and $p$. The behavior of the system described by Eq. (1) on the underlying network is very rich and one can have steady, periodic or chaotic states as well as fragmentation. In the following we analyze in more detail the system's phase diagram as well as how the dynamical regimes couple to the local structural properties of the underlying network and dynamical islands.

\section{A. Density distribution functions of dynamical regimes}

In Fig. 8, we have represented the probability, $P_{\text {chaos }}$, of ending up in a chaotic regime as a function of $p$ for a network of $N=300$ nodes and $h=4$. This probability is given by the fraction of the total number of realizations (typically $10^{3}$ different initial conditions over different network realizations for each value of $p$ and $h$ were used) in which at least one chaotic dynamics is observed. Figure 8 also shows the corresponding probability, $P_{\text {per }}$, for periodic orbits. As Fig. 8(a) clearly shows, there is a threshold value $p=p_{\text {th }}$ beyond which the network dynamics is not robust under variations of the initial values of the $g_{i}$ 's. For values of $p$ above $p_{\text {th }}$ $\approx 0.25(5)$, two randomly chosen initial conditions can lead the system to disparate asymptotic regimes. Besides, the size of the system affects the value of $P_{\text {chaos }}$, but the onset-and the end - of the chaotic phase seems to be $N$ independent. ${ }^{15}$

Moreover, Fig. 8(a) constitutes a quantitative illustration of how the prevalence of fluctuating asymptotic regimes over chemo-stasis ones depends on the model parameter $p$. The sum of both functions, $P_{\mathrm{per}}(p)+P_{\text {chaos }}(p)$, gives the probability that the asymptotic state shows temporal variations of the activity vector (either regular or chaotic) as a function of $p$. These results give that in the range of values $0.5 \leqslant p \leqslant 0.8$ regimes of temporal fluctuations occur more often than constant activity regimes. This measure is maximized by values around $p \simeq 2 / 3$ and, quite naturally, it increases with the value of the Michaelis-Menten parameter $h$, i.e., the slope at the origin of the saturated response function (see Fig. 1). Note that even larger values of $p$ means overabundance of inhibitory interactions, which leads to the predominance of the asymptotic rest state, while smaller values of $p$ favor chemostatic equilibria.

The quantities $P_{\text {chaos }}$ and $p_{\text {th }}$ depend on $h$. As we move to larger values of $h$, the strength of the interactions increases and hence it is expected that slight perturbations produce a behavior in which the fraction of nodes whose dynamical patterns are easily disturbed grows. This is indeed the case, as illustrated in Fig. 8(b). The color-coded figure shows that as $h$ is increased, the probability of having a chaotic phase grows, and that the onset of such chaotic patterns shifts to smaller values of $p$. This drift of $p_{\text {th }}$ is however bounded. For small enough values of $p$ (even for very large $h$ ), most of the elements activate each other $\left(W_{i j}=1\right.$ for a large fraction of pairs $i j$ and $j i$ ) and hence the resulting dynamics is steady. In other words, the onset of chaotic regimes is always located at a nonzero value of $p_{\text {th }}$ (the same applies to the right (decaying) part of $P_{\mathrm{ch}}(p)$, but in this case the activity falls down to zero).

\section{B. Dynamical island structure}

We next focus on the topological characterization of islands of dynamical units. We first analyze how the cluster size distribution of islands of nodes displaying either periodic or chaotic activity scales with the system size. Figure 9(a) represents the probability that an island has a given size for several networks made up of a number of nodes ranging from 50 to 800 . Clearly, the size distribution shows an average value that changes as $N$ grows. A closer look at the figure [see Fig. 9(b)] reveals that the mean cluster size scales with $N$ and that about $17 \%$ of the nodes, on average, exhibits 

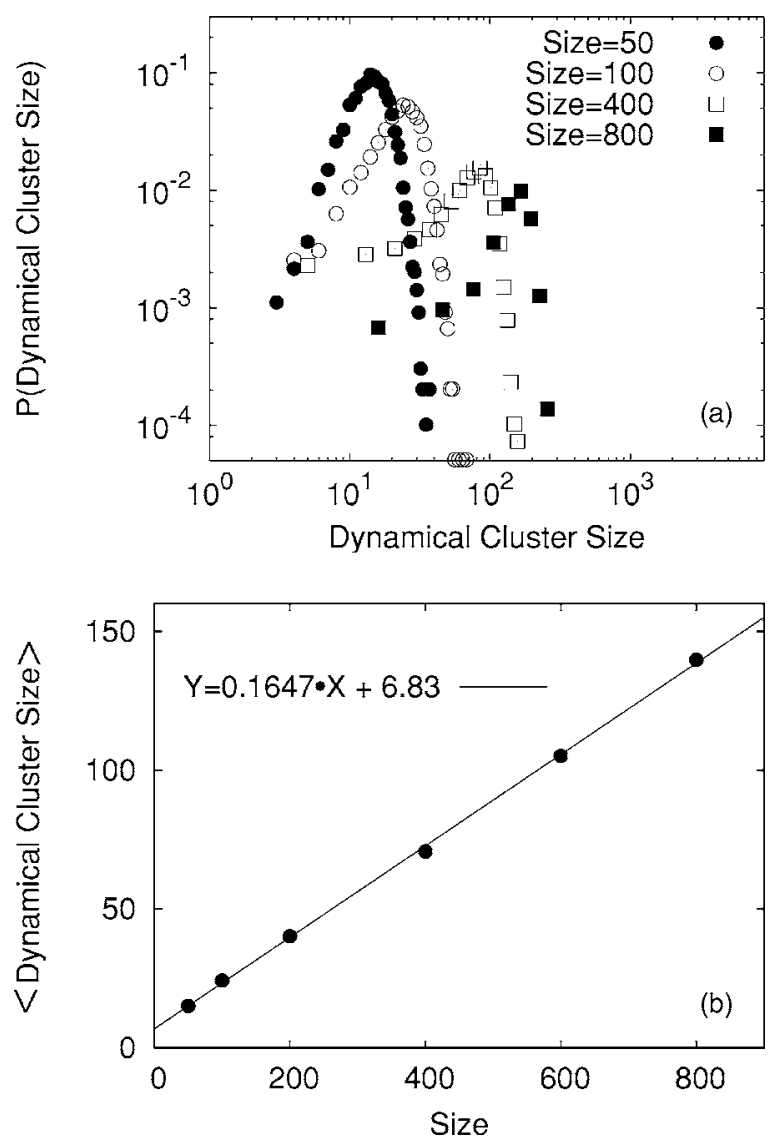

FIG. 9. (a) Probability that a connected cluster of nodes displaying either chaotic or periodic behavior has a given size (in number of nodes forming the cluster). (b) Scaling of the mean cluster size with $N$. The parameters have been set to $h=4$ and $p=0.7$.

nonzero activity. This confirms what we have discussed in Sec. II A about the measures of the sets $\mathcal{D}^{*}$ and $\mathcal{D}$, namely, that the fragmentation of the network into islands of independent dynamics appears as one of the most characteristic features of the model.

Another interesting aspect is the elucidation of how the topological properties of the islands correlate with those of the underlying (original) network. To this end, we have further scrutinized the structure of the clusters and measured two topological quantities of interest. Figure 10 shows the degree distribution of nodes belonging to dynamical islands for several system sizes. This property can be regarded as a global one and indicates that within the islands, the probability that a node has $k$ links also follows a power-law, though with a more pronounced cut-off and a (slightly) different value for the exponent $\gamma$. More striking is the result depicted in Fig. 11, where the average clustering coefficient $\langle C\rangle$ of the substrate (original) network and of the islands is plotted as a function of $N$. While for the BA network the clustering is vanishing as the network size grows, it seems that for dynamical islands its value saturates. This is quite interesting because, on the other hand, the value of the clustering coefficient is very large and comparable to measures of real systems where the kind of dynamics explored here applies, for instance, biological networks. ${ }^{17}$

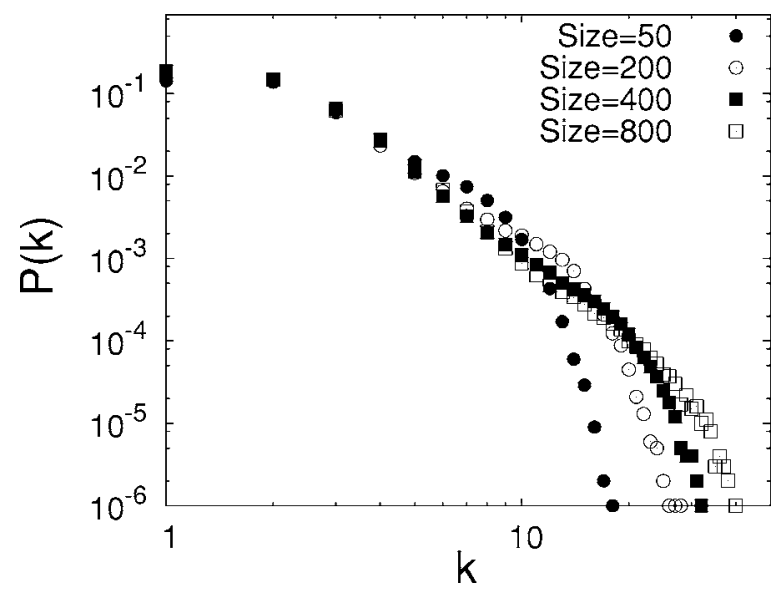

FIG. 10. Probability that a node belonging to a dynamical island interacts with $k$ other nodes of the island. Parameters were set to $h=4$ and $p=0.7$.

That is, the structure of dynamical islands correctly reproduces several of the most important topological features observed in biological networks and not captured by current network models. Namely, the heterogeneous distribution of connections, a high average clustering and the independence of $\langle C\rangle$ with respect to the system size. This result points to the conjecture that several topological properties observed in systems driven by AI interactions where nodes are themselves (nonlinear) dynamical units may be biased by their own dynamics. In other words, what we actually see is the result of the activity showed up by a smaller "dynamical" network whose local topological properties greatly differ from those of a larger substrate network that we do not "see" because many of its components are simply off. This, in fact, may be the case of biological systems where structure and dynamics are indissoluble linked. ${ }^{5,17}$

\section{Structure inside dynamical islands}

The above-presented findings on new (dynamically) emergent characteristics of the islands' structure motivates the question of whether these clusters have an internal orga-

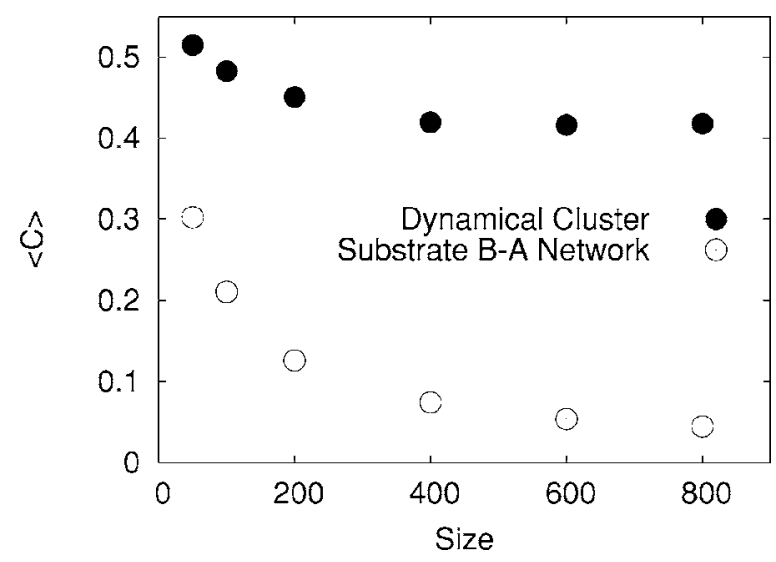

FIG. 11. Average clustering coefficient $\langle C\rangle$ as a function of the network size for the BA original network and the dynamical cluster. Note that while $\langle C\rangle$ in the BA network continuously decreases, for the dynamical island it saturates. See more details in the text. The results have been obtained using $h$ $=4$ and $p=0.7$. 

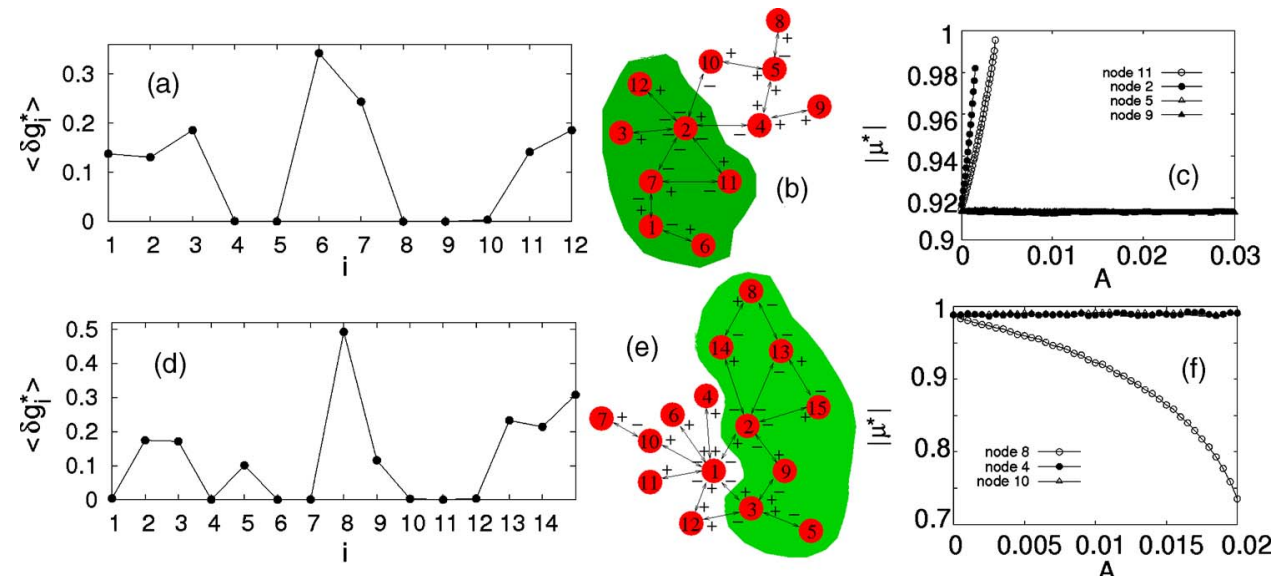

(e)
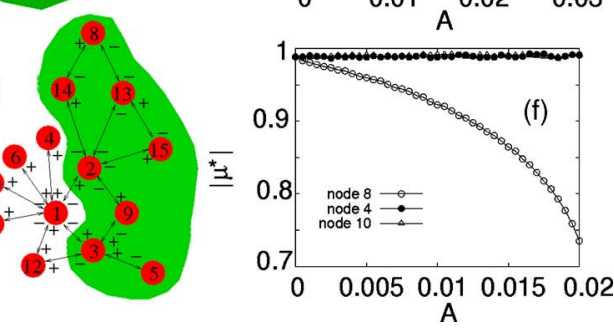

FIG. 12. (Color online) (a) and (d) The components of the vector $\left\langle\delta \vec{g}^{\star}\right\rangle$ (see the text) for two dynamical islands at the critical point of a Naimark-Sacker bifurcation at $h$ $=2.44$ (a) and a period doubling one at $h=2.629$ (d). (b) and (e) The distribution (green region) of the nodes with non-null component of $\left\langle\delta \vec{g}^{\star}\right\rangle$ in (a) and (d), respectively. Finally, (c) and (f) show the evolution of the Floquet eigenvalue $\mu^{\star}$ as a function of the forcing amplitude $A$ applied to different nodes of the dynamical islands (b) and (e), respectively. For both islands the substrate network was of $N=60$ nodes with a fraction of $80 \%$ of inhibitory interactions $(p=0.8)$. nization or hierarchy among its constituents. It is widely known that when one deals with problems where the network topology (scale-free) is the only degree of complexity of the problem the answer to this question is usually based on the presence of highly connected nodes (the hubs). This is the case when linear evolution equations are studied on top of complex networks like epidemic or rumor spreading, traffic, and communication problems. ${ }^{18-20}$ However, our case is not so simple and the nonlinear excitatory/inhibitory dynamics between the elements of the network plays a crucial role in determining which nodes are governing the evolution of the system. Moreover, the high clustering found for the dynamical clusters points out that this leading role is not played by isolated nodes but by small substructures inside the dynamical islands. This concept is not new, the problem of finding small relevant substructures inside large networks, usually called "motifs," 21 has been studied in different ways in the field of biological networks.

It is indeed very revealing to pay attention to the networked structure of the unstable manifold, which is given in the linear regime of small perturbations by the Floquet unstable eigenvectors. For this purpose, we look at the behavior of the components of the dynamical islands when a bifurcation (either period doubling or Naimark-Sacker type) occurs. In these critical points, it is possible to get a deeper insight into what is going on in the dynamical islands $I$ by looking at the Floquet eigenvector responsible for the bifurcation, $\delta \vec{g}^{\star}\left(t_{0}\right)=\left\{\delta g_{i}^{\star}\left(t_{0}\right)\right\}$, corresponding to the Floquet eigenvalue which reaches the unit circle. In particular, integrating Eq. (3) for the initial condition $\delta \vec{g}^{\star}\left(t_{0}\right)$ we can compute the following vector:

$$
\left\langle\overrightarrow{\delta g^{\star}}\right\rangle=\left\{\left\langle\delta g_{i}^{\star}\right\rangle\right\}=\left\{\frac{1}{T} \int_{0}^{T}\left|\delta g_{i}^{\star}\right| d t\right\} .
$$

The components of this vector measure, for each node, the average (over a period $T$ of the old solution) distance of the new solution after the bifurcation point from the old periodic solution. Note that a zero component of this vector at a node $k$ means orthogonality of the single-site perturbations at that node with respect to the unstable direction in tangent space. In other words, by looking at the components of the vector (6) we can identify those nodes that are more affected by the perturbation that leads the system to instability. In Figs. 12(a) and 12(d) we show this quantity for the same two islands (relatively small, but still representative) whose Floquet spectra are given in Fig. 7, one (a) corresponding to a Naimark-Sacker bifurcation and the other (d) to a period doubling bifurcation.

As can be seen from the figures, the vectors $\left\langle\delta \vec{g}^{\star}\right\rangle$ have several null components. The structural profiles reveal, apparently irrespective of the type of instability, that the set $\mathcal{S}$ of nodes in the island which are alien to instability (white regions), that is, the set of those nodes $k$ such that $\left\langle\delta g_{k}\right\rangle=0$, is a nonzero measure set; it is sometimes even larger than the complementary set (green area) $\mathcal{U}=I-\mathcal{S}$ of participating nodes on the unstable eigenvector evolution during a period. We observe here that the fragmentation tendency (see the earlier discussion on islands of disconnected dynamics) operates also at the level of the tangent space, in the sense that a binary partition of the island nodes is well defined at the bifurcation (critical) point. Namely, the instability introduces a partition of the island $I=\mathcal{U} \oplus \mathcal{S}$ into (a) the set $\mathcal{U}$ of nodes that do participate in the instability evolution in the linear regime, and (b) the complementary set $\mathcal{S}$ of nodes such that single-node perturbations are orthogonal to the unstable linear manifold. This drastic, generic fragmentation of the island of periodic activity at the linear description level of the bifurcation is also clearly the consequence of the AI competition on the network of interactions, and we have not seen any deviation from this observation in the computations performed (of which only two cases are illustrated). In summary, one could say that inside the dynamical islands there are compact substructures (and not single nodes) governing the dynamical changes of the whole cluster of nodes.

The above-described behavior suggests the following numerical experiment: we have explored the responses of the different nodes to an external perturbation when the system is in a periodic state near a bifurcation point. In particular, we force a node by adding an aditional term of the form $A \cdot \sin (\omega t)$ (with $\omega=2 \pi / T$ where $T$ the period of the unperturbed system) to its equation of motion (1). Then we compute, as a function of the forcing amplitude $A$, the evolution of the Floquet eigenvalue $\mu^{\star}$ responsible for the forthcoming bifurcation in the unperturbed system. The effects of such a 
perturbation strongly depend on whether the perturbed node belongs to the subset of those identified as leaders (i.e., the ones with non-null component in $\left.\left\langle\delta \vec{g}^{\star}\right\rangle\right)$. The results obtained for the two dynamical islands aforediscussed are shown in Figs. 12(c) and 12(f). When the nodes inside the green area are perturbed the Floquet eigenvalue $\mu^{\star}$ significantly deviate (either increase or decrease, we have not been able to elucidate when a given change is expected) from the values of the unperturbed system. On the other hand, the perturbation of the nodes located outside the green region does not imply any change to linear stability of the whole system. These results illustrate the relevant role played by the substructures found by the computation of $\left\langle\delta \vec{g}^{\star}\right\rangle$.

\section{CONCLUDING REMARKS}

In this paper, we have analyzed the interplay between complex topologies and activatory-inhibitory interactions driven by a saturated response dynamics of the MichaelisMenten type. The dynamics of the system is very rich and exhibits steady, periodic and chaotic regimes that in turn lead to the fragmentation of the original substrate network into a smaller cluster of dynamically active nodes. We have fully characterized these states by means of the Lyapunov exponent and the Floquet analyses as well as the topological features of active islands. The reach behavioral repertoire observed is thus a consequence of the entangled complexity of the system temporal behavior and the heterogeneous structure of the underlying network.

The emerging dynamics characterized in this work could plausibly describe at least two relevant scenarios in biological systems. On one hand, the dynamics expressed in Eq. (1) has been proposed as a way to characterize theoretically the individual dynamics of gene expression. ${ }^{13}$ In fact, Eq. (1) is the generalization of the successful random Boolean models ${ }^{11,12}$ widely used to model gene expression. In this context, two nodes at the ends of a link are considered to be transcriptional units which include a regulatory gene. One of these end-nodes can be thought of as being the source of an interaction (the output of a transcriptional unit). The second node represents the target binding site and at the same time the input of a second transcriptional unit. By studying simplified models as the one implemented here-the intrinsic complexity of the problem does not allow for a complete and detailed description of real gene dynamics-one can infer the region of the parameter space [i.e., $(p, h)]$ that better describes gene networks. The latter seems possible due to latest developments in microarray technologies, biocomputational tools, and data collection software.

A second scenario where the results obtained apply is reaction kinetics in metabolic networks. In metabolic systems, a very rich behavioral repertoire is well documented, ${ }^{22}$ as for instance, the oscillations observed in the concentration of certain chemicals in biochemical reactions such as glycolysis. The system of differential equations, Eq. (1), represents one of the most basic biochemical reactions, where substrates and enzymes are involved in a reaction that produces a given product. In this context, there are several important issues as how fast the equilibrium is reached, how the con- centration of substrates and enzymes compare, etc. Besides, it is known that in a large number of situations, some of the enzymes involved show periodic increments in their activity during division, and these reflect periodic changes in the rate of enzyme synthesis. This is achieved by regulatory mechanisms that necessarily require some kind of feedback control as that emerging in our model. The interesting point here is that the real topological features of the underlying metabolic network $^{23}$ have not been taken into account in studies performed so far. As this work shows, they have important bearings in the correlation between structure and the observed dynamics.

Finally, on more general theoretical grounds, we anticipate several features of interest such as the fragmentation of the original network according to the dynamical states of the nodes, multistability and different routes to chaotic behavior within the same system. The first of these points is particularly relevant since it may indicate that in networks of dynamical units, the topology observed can be the result of a given network state hiding a larger substrate whose topological properties are completely different at a local level. Of particular interest is also the result gathered in the last part of the work, namely, the existence of an additional substructure inside dynamical islands determined by the different responses of nodes to external perturbations. This points to the central issue in many biological processes of what subset of nodes are the most important in order to sustain (or break) the system's robust functioning. In summary, the characterization of models where nonlinearity and spatial complexity coexist yields new results missed when only one of these ingredients is present and opens the path to a better comprehension of biological processes and the dynamics of networks of nonlinear dynamical units.

\section{ACKNOWLEDGMENTS}

The authors thank S. Boccaletti and S. Manrubia for useful suggestions and discussions. J.G.-G. acknowledges financial support of the MECyD through a FPU grant. Y.M. is supported by MEC through the Ramón y Cajal program. This work has been partially supported by the Spanish DGICYT Project Nos. BFM2002-00113, BFM2002-01798, and FIS2004-05073-C04-01; and by DGA (grupo consolidado FENOL).

\footnotetext{
${ }^{1}$ A. Scott, Nonlinear Science (Oxford University Press, Oxford, 1999).

${ }^{2}$ Encyclopedia of Nonlinear Science, edited by A. Scott (Routledge, New York, 2005).

${ }^{3}$ See, e.g., Handbook of Graphs and Networks: From the Genome to the Internet, edited by S. Bornholdt and H. G. Schuster (Wiley-VCH, Berlin, 2002).

${ }^{4}$ R. Albert and A.-L. Barabási, Rev. Mod. Phys. 74, 47 (2002).

${ }^{5}$ S. H. Strogatz, Nature (London) 410, 268 (2001).

${ }^{6}$ L. A. Segel, Modeling Dynamic Phenomena in Molecular and Cellular Biology (Cambridge University Press, Cambridge, 1984); L. Michaelis and L. M. Menten, Biochem. Z. 49, 333 (1913).

${ }^{7}$ B. Drosssel and A. J. McKane, in Handbook of Graphs and Networks: From the Genome to the Internet, edited by S. Bornholdt and H. G. Schuster (Wiley-VCH, Berlin, 2002); C. S. Holling, Mem. Ent. Soc. Can. 45, 1 (1965).

${ }^{8}$ B. Diu, C. Guthmann, D. Lederer, and B. Roulet, Physique Statistique (Hermann, Paris, 1989); I. Langmuir, J. Chem. Soc. 40, 1361 (1918).

${ }^{9}$ A.-L. Barabási and R. Albert, Science 286, 509 (1999); A.-L. Barabási, R.
} 
Albert, and H. Jeong, Physica A 272, 173 (1999).

${ }^{10} \mathrm{~S}$. N. Dorogotsev and J. F. F. Mendes, Evolution of Networks. From Biological Nets to the Internet and the WWW (Oxford University Press, Oxford, 2003).

${ }^{11}$ S. Kauffman, Origin of Order (Oxford University Press, Oxford, 1993); Physica A 340, 733 (2004).

${ }^{12}$ C. Oosawa and M. A. Savageau, Physica D 170, 143 (2002).

${ }^{13}$ R. V. Solé, I. Salazar-Ciudad, and J. Garcia-Fernández, Physica A 305, 640 (2002); R. V. Solé and R. Pastor-Satorras, in Handbook of Graphs and Networks: From the Genome to the Internet, edited by S. Bornholdt and $\mathrm{H}$. G. Schuster (Wiley-VCH, Berlin, 2002).

${ }^{14}$ T. S. Gardner et al., Science 301, 102 (2003).

${ }^{15}$ J. Gómez-Gardeñes, Y. Moreno, and L. M. Floría, Physica A 352, 265 (2005); Biophys. Chem. 115, 225 (2005).
${ }^{16}$ R. Seydel, Practical Bifurcation and Stability Analysis (Springer, New York, 1994).

${ }^{17}$ A.-L. Barabási and Z. N. Oltvai, Nat. Rev. Genet. 5, 101 (2004).

${ }^{18}$ Y. Moreno, M. Nekovee, and A. Vespignani, Phys. Rev. E 69, 055101(R) (2004).

${ }^{19} \mathrm{P}$. Echenique, J. Gómez-Gardeñes, and Y. Moreno, Europhys. Lett. 71, 325 (2005).

${ }^{20} \mathrm{P}$. Echenique, J. Gómez-Gardeñes, Y. Moreno, and A. Vázquez, Phys. Rev. E 71, 035102(R) (2005).

${ }^{21}$ R. Milo, S. Shen-Orr, S. Itzkovitz, N. Kashtan, D. Chklovskii, and U. Alon, Science 298, 824 (2002).

${ }^{22}$ J. D. Murray, Mathematical Biology I: An Introduction, 3rd ed. (Springer, Berlin, 2002).

${ }^{23}$ H. Jeong, B. Tombor, R. Albert, Z. Oltvai, and A.-L. Barabási, Nature (London) 407, 651 (2000). 